\title{
ASYMPTOTIC BEHAVIOUR OF STOCHASTIC SYSTEMS WITH CONDITIONALLY EXPONENTIAL DECAY PROPERTY
}

Abstract. A new class of CED systems, providing insight into behaviour of physical disordered materials, is introduced. It includes systems in which the conditionally exponential decay property can be attached to each entity. A limit theorem for the normalized minimum of a CED system is proved. Employing different stable schemes the universal characteristics of the behaviour of such systems are derived.

1. Introduction. Let $\left\{A_{i}: i=1,2, \ldots\right\}$ and $\left\{B_{j}^{i}: i, j=1,2, \ldots, j \neq\right.$ $i\}$ be two independent sequences of nonnegative independent identically distributed (i.i.d.) random variables (r.v.'s). Let the symbol " $\diamond$ " stand for one of the three operations: summation, minimum, or maximum, and let $n \in \mathbb{N}$, $\widetilde{b}_{n}, r, s>0$, and $c \geq 0$ be constants.

Definition. A sequence $X_{1 n}, \ldots, X_{n n}$ of independent r.v.'s is called the $C E D$ system given $\left\{A_{i}: i=1,2, \ldots\right\}$ and $\left\{B_{j}^{i}: i, j=1,2, \ldots, j \neq i\right\}$ with the operation $\diamond$ and the parameters $n \geq 2, \widetilde{b}_{n}, c, r$, and $s$ iff it has the conditionally exponential decay property, i.e., the conditional tails

$$
P\left(X_{i n} \geq x \mid A_{i}=a, \widetilde{b}_{n}\left(B_{1}^{i} \diamond \ldots \diamond B_{i-1}^{i} \diamond B_{i+1}^{i} \diamond \ldots \diamond B_{n}^{i}\right)=b\right)
$$

have for each $i=1, \ldots, n$ a common exponential decay form:

$$
1-G(x \mid a, b) \equiv \begin{cases}\exp \left(-a x^{r}\right) & \text { if } c=0 \\ \exp \left(-a \min \left(x^{r},(b / c)^{s}\right)\right) & \text { if } c>0\end{cases}
$$

for $a, b, x \geq 0$.

1991 Mathematics Subject Classification: Primary 82A57; Secondary 60E07, 82A31.

Key words and phrases: stochastic CED systems; stability of stochastic models; stable distributions; minima of random sequences; dielectric relaxation; reaction kinetics.

This work was partially supported by KBN grant No. 2115391 01, KBN grant No. 2 P302 065 04, and NSF grant No. INT 92-20285. 
Note that when the parameter $c$ is positive each r.v. $X_{i n}$, interpreted as an element of the CED system, is infinite with a nonzero probability, i.e., its distribution function (d.f.) is improper. The parameter $n$ simply indicates the number of elements in the system.

The right-hand side of formula (2) satisfies all requirements of the conditional tail (1) [Billingsley (1979)]. Namely, it is a Borel function with respect to $a$ and $b$, and is nonincreasing with respect to $x$. Moreover, it is nonnegative and at most 1 . It turns out that this suffices for construction of a sequence of CED systems given the same families of r.v.'s with the same operation $\diamond$ and the same parameters $c, r, s$.

Existence TheOREM. Let $F_{A}$ and $F_{B}$ be d.f.'s such that $F_{A}(0)=0$ and $F_{B}(0)=0$. Let $\left\{\widetilde{b}_{n}: n=2,3, \ldots\right\}$ be a sequence of positive constants, an operation $\diamond=+$, $\min$ or $\max , r, s>0$, and $c \geq 0$. On some probability space there exist:

- independent sequences $\left\{A_{i}: i=1,2, \ldots\right\}$ and $\left\{B_{j}^{i}: i, j=1,2, \ldots\right.$, $j \neq i\}$ of nonnegative i.i.d. r.v.'s such that $F_{A}$ and $F_{B}$ are d.f.'s of $A_{i}$ and $B_{j}^{i}$, respectively;

- and a sequence

$$
\begin{array}{cccc}
X_{12} & X_{22} & & \\
X_{13} & X_{23} & X_{33} & \\
\vdots & & & \\
X_{1 n} & X_{2 n} & \ldots & X_{n n}
\end{array}
$$

of CED systems, $n=2,3, \ldots$, given $\left\{A_{i}: i=1,2, \ldots\right\}$ and $\left\{B_{j}^{i}: i, j=\right.$ $1,2, \ldots, j \neq i\}$ with the operation $\diamond$ and the parameters $n, \widetilde{b}_{n}, c, r$, and $s$.

The above theorem allows us to define the asymptotic description of the behaviour of a CED system in Section 2, which leads to a new result for random variables which are infinite with a nonzero probability. The result, presented in Theorems 1 and 2, provides the universal characteristics of CED systems. Moreover, the differential equation obtained in Theorem 2 may be recognized as the most useful tool in statistical mechanics [Van Kampen (1987)], namely, a generalized master equation. Section 3 contains the proofs of all theorems formulated in this paper. In the last section we sketch the application of the presented approach to disordered systems in the case of dielectric relaxation in polar materials and in the analysis of nonexponential first-order chemical reactions.

2. Asymptotic behaviour of CED systems. The main idea of statistical physics [Van Kampen (1987)] assumes that the behaviour of any 
stochastic physical system as a whole is represented by an appropriate averaging over its elements. For CED systems the averaged minimum $\widetilde{m} \min \left(X_{1 n}, \ldots, X_{n n}\right), \widetilde{m}>0$, is proposed, which has been suggested by applications [Weron (1991)]. When the parameter $n$ is sufficiently large (i.e., the system consists of a very large number of elements) the behaviour of the system can be approximately described by the following limit in distribution:

$$
\mathbf{X}=\lim _{n \rightarrow \infty} \widetilde{m}_{n} \min \left(X_{1 n}, \ldots, X_{n n}\right)
$$

if it exists. Here $\widetilde{m}_{n}, n=2,3, \ldots$, are positive normalizing constants. Immediately, there arises a question if and under what assumptions the limiting r.v. $\mathbf{X}$ exists.

Theorem 1. Let $\left\{A_{i}: i=1,2, \ldots\right\}$ and $\left\{B_{j}^{i}: i, j=1,2, \ldots, j \neq i\right\}$ be independent sequences of nonnegative i.i.d. r.v.'s such that the d.f. of $B_{2}^{1}$ is continuously differentiable. Let $\diamond=+$, min, or $\max$, and $r, s>0$. Assume that for sequences of positive constants $\left\{\widetilde{b}_{n}: n=2,3, \ldots\right\}$ and $\left\{\widetilde{m}_{n}: n=2,3, \ldots\right\}$ the following nonzero limits in distribution exist:

$$
\mathbf{A}=\lim _{n \rightarrow \infty} \frac{A_{1}+\ldots+A_{n}}{\widetilde{m}_{n}^{r}}
$$

and

$$
\mathbf{B}=\lim _{n \rightarrow \infty} \widetilde{m}_{n}^{r / s} \widetilde{b}_{n}\left(B_{2}^{1} \diamond \ldots \diamond B_{n}^{1}\right) .
$$

Additionally, let the limits $\mathbf{A}$ and $\mathbf{B}$ be finite with probability 1 .

For some $c \geq 0$ consider a sequence (3) of CED systems, $n=2,3, \ldots$, given $\left\{A_{i}: i=1,2, \ldots\right\}$ and $\left\{B_{j}^{i}: i, j=1,2, \ldots, j \neq i\right\}$ with the operation $\diamond$ and parameters $n, \widetilde{b}_{n}, c, r$, and $s$. Then the nondegenerate limit in distribution

$$
\mathbf{X}=\lim _{n \rightarrow \infty} \tilde{m}_{n} \min \left(X_{1 n}, \ldots, X_{n n}\right)
$$

exists. Moreover, the d.f. $\mathbf{F}$ of $\mathbf{X}$ has the form

$$
\mathbf{F}(x)=1-\exp \left(\int_{0}^{x}\left(1-\mathbf{F}_{B}\left(c u^{r / s}\right)\right) \frac{d}{d u} \ln \mathcal{L}\left(\mathbf{F}_{A} ; u^{r}\right) d u\right),
$$

where $\mathbf{F}_{A}$ and $\mathbf{F}_{B}$ are the d.f.'s of $\mathbf{A}$ and $\mathbf{B}$, respectively, and $\mathcal{L}(F ; \cdot)$ denotes the Laplace transform of a d.f. $F$.

The existence of the limiting random properties $\mathbf{A}$ and $\mathbf{B}$ (see (5) and (6)) is therefore a sufficient condition for the possibility of asymptotic characterization of the behaviour of the CED system by the r.v. X, given by (4). Moreover, the above theorem gives us the connections between the d.f.'s of $\mathbf{A}, \mathbf{B}$, and $\mathbf{X}$. To specify the form of $\mathbf{F}$ (see (7)), let us first focus our attention on the $\diamond$-stability property. Although the stability concept associated 
with operations over the set of i.i.d. r.v.'s can be introduced in the general case, we restrict ourselves to nonnegative r.v.'s.

Let $Z_{1}, Z_{2}, \ldots$ be nonnegative nondegenerate i.i.d. r.v.'s which are finite with probability 1 . The distribution of $Z_{1}$ is $\diamond$-stable iff for any $n$ there exists a positive constant $\widetilde{a}_{n}$ such that

$$
Z_{1} \stackrel{d}{=} \widetilde{a}_{n}\left(Z_{1} \diamond \ldots \diamond Z_{n}\right),
$$

where " $=$ " denotes equality in distribution. It is known that the standard summation scheme produces nonnegative $\alpha$-stable distributions [Feller (1966), Zolotarev (1986), Janicki and Weron (1994)], and the maximum and minimum schemes lead to nonnegative extreme-value laws [Leadbetter, Lindgren and Rootzen (1986)]. For other possible stable schemes, see Mittnik and Rachev (1991), Rachev (1991). The important property of stable distributions is that they have domains of attraction. Namely, for nonnegative i.i.d. r.v.'s $Z_{1}, Z_{2}, \ldots$ which are finite with probability 1 , if the nondegenerate limit in distribution $Z=\lim _{n \rightarrow \infty} \widetilde{a}_{n}\left(Z_{1} \diamond \ldots \diamond Z_{n}\right)$ exists then it is $\diamond$-stable. (Here $\widetilde{a}_{n}, n=1,2, \ldots$, are positive normalizing constants.)

For convenience we collect in the following table the forms of the d.f.'s $F_{\diamond}$ of $\diamond$-stable laws considered in the present paper $(\diamond=+$, min, and max).

\begin{tabular}{lcl}
\hline$\diamond$ & Stable scheme & Form of $F_{\diamond}$ \\
\hline \hline+ & $Z_{1} \stackrel{d}{=} \widetilde{a}_{n}\left(Z_{1}+\ldots+Z_{n}\right)$ & $\begin{array}{l}\mathcal{L}\left(F_{\diamond} ; x\right)=\exp \left(-(\Lambda x)^{\alpha}\right), x \geq 0, \\
\text { for some } 0<\alpha<1, \Lambda>0\end{array}$ \\
\hline $\min$ & $Z_{1} \stackrel{d}{=} \widetilde{a}_{n} \min \left(Z_{1}, \ldots Z_{n}\right)$ & $\begin{array}{l}F_{\diamond}(x)=1-\exp \left(-(\Lambda x)^{\gamma}\right), x>0, \\
\text { for some } \gamma>0, \Lambda>0\end{array}$ \\
\hline $\max$ & $Z_{1} \stackrel{d}{=} \widetilde{a}_{n} \max \left(Z_{1}, \ldots Z_{n}\right)$ & $\begin{array}{l}F_{\diamond}(x)=\exp \left(-(\Lambda x)^{-\gamma}\right), x>0, \\
\text { for some } \gamma>0, \Lambda>0\end{array}$ \\
\hline
\end{tabular}

From the above stable schemes it follows that the d.f.'s $\mathbf{F}_{A}$ and $\mathbf{F}_{B}$ take on specific forms. This leads to:

THEOREM 2. Under the assumptions of Theorem 1:

- When $c=0$ the limiting d.f. $\mathbf{F}$, given by (7), has the form

$$
\mathbf{F}(x)=1-\exp \left(-\left(\Lambda_{1} x^{r}\right)^{\alpha}\right) .
$$

- When $c>0$ there are two possible types of the d.f. $\mathbf{F}$ :

$$
\mathbf{F}(x)=1-\exp \left(-\left(\Lambda_{1} \min \left(x^{r},\left(b_{0} / c\right)^{s}\right)\right)^{\alpha}\right)
$$

and the solution of the differential equation

$$
\frac{d \mathbf{F}}{d x}(x)=\alpha r \Lambda_{1}^{\alpha} x^{r \alpha-1}\left(1-\mathbf{F}_{B}\left(c x^{r / s}\right)\right)(1-\mathbf{F}(x)), \quad \mathbf{F}(0)=0,
$$


where:

$$
\begin{aligned}
& \mathcal{L}\left(\mathbf{F}_{B} ; x\right)=\exp \left(-\left(\Lambda_{2} x\right)^{\gamma}\right) \quad \text { for some } 0<\gamma<1, \Lambda_{2}>0 \text { if } \diamond=+; \\
& \text { (10) } \quad \mathbf{F}_{B}(x)=1-\exp \left(-\left(\Lambda_{2} x\right)^{\gamma}\right) \quad \text { for some } \gamma>0, \Lambda_{2}>0 \text { if } \diamond=\min \text {; } \\
& \mathbf{F}_{B}(x)=\exp \left(-\left(\Lambda_{2} x\right)^{-\gamma}\right) \quad \text { for some } \gamma>0, \Lambda_{2}>0 \text { if } \diamond=\max .
\end{aligned}
$$

In all cases, $x \geq 0,0<\alpha \leq 1$, and $\Lambda_{1}$ is a positive constant.

\section{Proofs}

Proof of the Existence Theorem. To prove the existence theorem we construct first a probability space $(\Omega, \mathcal{B}, P)$. Define $\overline{\mathbb{R}}_{+}=\mathbb{R}_{+} \cup$ $\{+\infty\}$. Let

and for $n>1$,

$$
\Omega_{1}=\mathbb{R}_{+}=\left\{a_{1} \in \mathbb{R}_{+}\right\}
$$

$$
\begin{aligned}
\Omega_{n} & =\mathbb{R}_{+}^{2 n-1} \times \overline{\mathbb{R}}_{+}^{n} \\
& =\left\{\left(a_{n}, b_{n}^{1}, \ldots, b_{n}^{n-1}, b_{1}^{n}, \ldots, b_{n-1}^{n}, x_{1 n}, \ldots, x_{n n}\right) \in \mathbb{R}_{+}^{2 n-1} \times \overline{\mathbb{R}}_{+}^{n}\right\}
\end{aligned}
$$

be measurable spaces with the corresponding Borel $\sigma$-fields $\mathcal{B}_{n}$. Since $G(x \mid a, b)$ (see $(2))$ as a function of $x$ is a d.f., improper in case $c>0$, it defines a probability measure $\mu(\cdot \mid a, b)$ on $\overline{\mathbb{R}}_{+}$for any fixed $a, b \in \mathbb{R}_{+}$. Namely, for a Borel subset $C$ of $\mathbb{R}_{+}$,

$$
\mu(C \mid a, b)=\int_{C} d G(x \mid a, b)
$$

and

$$
\mu(\{+\infty\} \mid a, b)=1-\int_{0}^{\infty} d G(x \mid a, b) .
$$

Then the probability measure $P_{n}$ on the product space $\left(\prod_{k=1}^{n} \Omega_{k}, \prod_{k=1}^{n} \mathcal{B}_{k}\right)$ given by

$$
\begin{aligned}
& (13) \quad P_{n}(C) \\
& =\iint_{\mathbb{R}_{+}} \ldots \int_{\mathbb{R}_{+}}\left\{\int_{\overline{\mathbb{R}}_{+}} \ldots \int_{\overline{\mathbb{R}}_{+}} \mathbf{1}_{C} d \mu\left(x_{12} \mid a_{1}, \widetilde{b}_{1} b_{2}^{1}\right) \ldots d \mu\left(x_{1 n} \mid a_{1}, \widetilde{b}_{n}\left(b_{2}^{1} \diamond \ldots \diamond b_{n}^{1}\right)\right)\right. \\
& \left.\ldots d \mu\left(x_{n n} \mid a_{n}, \widetilde{b}_{n}\left(b_{1}^{n} \diamond \ldots \diamond b_{n-1}^{n}\right)\right)\right\} d F_{A}\left(a_{1}\right) \ldots \\
& \ldots d F_{A}\left(a_{n}\right) d F_{B}\left(b_{2}^{1}\right) \ldots d F_{B}\left(b_{n-1}^{n}\right)
\end{aligned}
$$

is well defined [Billingsley (1979)]. Moreover, the family $\left\{P_{n}: n=1,2, \ldots\right\}$ is consistent. Therefore, it follows from the Kolmogorov extension theorem [Breiman (1992)] that there exists a probability measure $P$ on the infinite 
product space $(\Omega, \mathcal{B})$, where $\Omega=\prod_{n=1}^{\infty} \Omega_{n}$ and $\mathcal{B}=\prod_{n=1}^{\infty} \mathcal{B}_{n}$, such that

$$
\left.P\right|_{\Pi_{k=1}^{n} \mathcal{B}_{k}}=P_{n} .
$$

It will now be shown that the projections, defined for $\omega \in \Omega$,

$$
\begin{aligned}
A_{i}(\omega) & =a_{i}, & & i=1,2, \ldots, \\
B_{j}^{i}(\omega) & =b_{j}^{i}, & & i, j=1,2, \ldots, i \neq j, \\
X_{i n}(\omega) & =x_{i n}, & & n=2,3, \ldots, i=1, \ldots, n,
\end{aligned}
$$

\begin{tabular}{|c|c|c|c|c|c|}
\hline Space & & Proj & ections & & \\
\hline$\Omega_{1}$ & $A_{1}$ & & & & \\
\hline$\Omega_{2}$ & $B_{2}^{1}$ & $A_{2}, B_{1}^{2}$ & & & \\
\hline$\Omega_{3}$ & $\begin{array}{c}X_{12} \\
B_{3}^{1} \\
X_{13}\end{array}$ & $\begin{array}{c}X_{22} \\
B_{3}^{2} \\
X_{23}\end{array}$ & $\begin{array}{c}A_{3}, B_{1}^{3}, B_{2}^{3} \\
X_{33}\end{array}$ & & \\
\hline $\begin{array}{c}\vdots \\
\Omega_{n}\end{array}$ & $\begin{array}{c}\vdots \\
B_{n}^{1} \\
X_{1 n}\end{array}$ & $\begin{array}{c}B_{n}^{2} \\
X_{2 n}\end{array}$ & $\begin{array}{c}B_{n}^{3} \\
X_{3 n}\end{array}$ & $\begin{array}{lc}\ldots & B_{n}^{n-1} \\
\ldots & X_{n-1, n}\end{array}$ & $\begin{array}{c}A_{n}, B_{1}^{n}, \ldots B_{n-1}^{n} \\
X_{n n}\end{array}$ \\
\hline$\vdots$ & $\vdots$ & & & & \\
\hline
\end{tabular}

are r.v.'s satisfying the conclusion of the existence theorem. Note that the projections (15) are directly related to the spaces $\Omega_{n}, n=1,2, \ldots$, which is illustrated in the following table:

For finite subsets $S_{1} \subset \mathbb{N}$ and $S_{2} \subset\{(j, k) \in \mathbb{N} \times \mathbb{N}: j \neq k\}$ let $m$ denote the greater of $\max S_{1}$ and $\max \left\{\max (j, k):(j, k) \in S_{2}\right\}$. For any positive constants $\widetilde{a}_{i}, i \in S_{1}$, and $\widetilde{b}_{j}^{k},(j, k) \in S_{2}$, it follows from (14) and then from (11)-(13) that

$$
\begin{aligned}
P\left(A_{i} \leq \widetilde{a}_{i}, i \in S_{1}, B_{j}^{k}\right. & \left.\leq \widetilde{b}_{j}^{k},(j, k) \in S_{2}\right) \\
& =P_{m}\left(A_{i} \leq \widetilde{a}_{i}, i \in S_{1}, B_{j}^{k} \leq \widetilde{b}_{j}^{k},(j, k) \in S_{2}\right) \\
& =\prod_{i \in S_{1}} F_{A}\left(\widetilde{a}_{i}\right) \prod_{(j, k) \in S_{2}} F_{B}\left(\widetilde{b}_{j}^{k}\right) .
\end{aligned}
$$

Hence, for any $i, j \in \mathbb{N}, i \neq j$, the functions $F_{A}$ and $F_{B}$ are d.f.'s of $A_{i}$ and $B_{j}^{i}$, respectively, and $A_{i}, i=1,2, \ldots$ and $B_{j}^{i}, i, j=1,2, \ldots, i \neq j$, form independent sequences of nonnegative i.i.d. r.v.'s.

To show that for the projections $X_{i n}, A_{i}, B_{1}^{i}, \ldots, B_{i-1}^{i}, B_{i+1}^{i}, \ldots, B_{n}^{i}$ the conditional tail (1) has the form (2) for each $n \in \mathbb{N}$ and $1 \leq i \leq n$ it is enough to prove that for any subset $C=\left\{A_{i} \in C_{1}, \widetilde{b}_{n}\left(B_{1}^{i} \diamond \ldots \diamond B_{i-1}^{i} \diamond\right.\right.$ $\left.\left.B_{i+1}^{i} \diamond \ldots \diamond B_{n}^{i}\right) \in C_{2}\right\}$, where $C_{1}, C_{2}$ are Borel subsets of $\mathbb{R}_{+}$, we have 
(16) $P\left(\left\{X_{\text {in }} \geq x\right\} \cap C\right)$

$$
=\int_{C}\left\{1-G\left(x \mid A_{i}, \widetilde{b}_{n}\left(B_{1}^{i} \diamond \ldots \diamond B_{i-1}^{i} \diamond B_{i+1}^{i} \diamond \ldots \diamond B_{n}^{i}\right)\right)\right\} d P .
$$

From (11)-(14),

$P\left(\left\{X_{\text {in }} \geq x\right\} \cap C\right)$

$$
\begin{aligned}
= & P_{n}\left(X_{i n} \geq x, A_{i} \in C_{1}, \widetilde{b}_{n}\left(B_{1}^{i} \diamond \ldots \diamond B_{i-1}^{i} \diamond B_{i+1}^{i} \diamond \ldots \diamond B_{n}^{i}\right) \in C_{2}\right) \\
= & \int_{\mathbb{R}_{+}} \ldots \int_{\mathbb{R}_{+}} \mathbf{1}_{C_{1}}\left(a_{i}\right) \mathbf{1}_{C_{2}}\left(\widetilde{b}_{n}\left(b_{1}^{i} \diamond \ldots \diamond b_{i-1}^{i} \diamond b_{i+1}^{i} \diamond \ldots \diamond b_{n}^{i}\right)\right) \\
& \times\left\{\int_{\mathbb{R}_{+}} \mathbf{1}_{[x, \infty]}\left(x_{i n}\right) d \mu\left(x_{i n} \mid a_{i}, \widetilde{b}_{n}\left(b_{1}^{i} \diamond \ldots \diamond b_{i-1}^{i} \diamond b_{i+1}^{i} \diamond \ldots \diamond b_{n}^{i}\right)\right)\right\} \\
& \times d F_{A}\left(a_{i}\right) d F_{B}\left(b_{1}^{i}\right) \ldots d F_{B}\left(b_{i-1}^{i}\right) d F_{B}\left(b_{i+1}^{i}\right) \ldots d F_{B}\left(b_{n}^{i}\right) .
\end{aligned}
$$

For any fixed $a, b \in \mathbb{R}_{+},(11)$ and (12) give us

$$
\int_{\overline{\mathbb{R}}_{+}} \mathbf{1}_{[x, \infty]}\left(x_{i n}\right) d \mu\left(x_{i n} \mid a, b\right)=1-G\left(x_{i n} \mid a, b\right) .
$$

Therefore

(17) $P\left(\left\{X_{\text {in }} \geq x\right\} \cap C\right)$

$$
\begin{aligned}
= & \int_{\mathbb{R}_{+}} \ldots \int_{\mathbb{R}_{+}} \mathbf{1}_{C_{1}}\left(a_{i}\right) \mathbf{1}_{C_{2}}\left(\widetilde{b}_{n}\left(b_{1}^{i} \diamond \ldots \diamond b_{i-1}^{i} \diamond b_{i+1}^{i} \diamond \ldots \diamond b_{n}^{i}\right)\right) \\
& \times\left\{1-G\left(x_{i n} \mid a_{i}, \widetilde{b}_{n}\left(b_{1}^{i} \diamond \ldots \diamond b_{i-1}^{i} \diamond b_{i+1}^{i} \diamond \ldots \diamond b_{n}^{i}\right)\right)\right\} \\
& \times d F_{A}\left(a_{i}\right) d F_{B}\left(b_{1}^{i}\right) \ldots d F_{B}\left(b_{i-1}^{i}\right) d F_{B}\left(b_{i+1}^{i}\right) \ldots d F_{B}\left(b_{n}^{i}\right) .
\end{aligned}
$$

On the other hand,

(18)

$$
\begin{aligned}
\int_{C}\left\{1-G\left(x \mid A_{i}, \widetilde{b}_{n}\left(B_{1}^{i} \diamond \ldots \diamond B_{i-1}^{i} \diamond B_{i+1}^{i} \diamond \ldots \diamond B_{n}^{i}\right)\right)\right\} d P \\
=\int_{C}\left\{1-G\left(x \mid A_{i}, \widetilde{b}_{n}\left(B_{1}^{i} \diamond \ldots \diamond B_{i-1}^{i} \diamond B_{i+1}^{i} \diamond \ldots \diamond B_{n}^{i}\right)\right)\right\} d P_{n} \\
=\int_{\mathbb{R}_{+}} \ldots \int_{\mathbb{R}_{+}} \mathbf{1}_{C_{1}}\left(a_{i}\right) \mathbf{1}_{C_{2}}\left(\widetilde{b}_{n}\left(b_{1}^{i} \diamond \ldots \diamond b_{i-1}^{i} \diamond b_{i+1}^{i} \diamond \ldots \diamond b_{n}^{i}\right)\right) \\
\quad \times\left\{1-G\left(x_{i n} \mid a_{i}, \widetilde{b}_{n}\left(b_{1}^{i} \diamond \ldots \diamond b_{i-1}^{i} \diamond b_{i+1}^{i} \diamond \ldots \diamond b_{n}^{i}\right)\right)\right\} \\
\quad \times d F_{A}\left(a_{i}\right) d F_{B}\left(b_{1}^{i}\right) \ldots d F_{B}\left(b_{i-1}^{i}\right) d F_{B}\left(b_{i+1}^{i}\right) \ldots d F_{B}\left(b_{n}^{i}\right) .
\end{aligned}
$$

Comparing the results (17) and (18) we obtain (16), which completes the proof. 
Proof of Theorem 1. In CED systems the form of the conditional tail (1) does not depend on $i$ (see (2)). Hence, the independent r.v.'s $X_{1 n}, \ldots, X_{n n}$ are identically distributed and we have

$$
P\left(\widetilde{m}_{n} \min \left(X_{1 n}, \ldots, X_{n n}\right) \geq x\right)=\left(P\left(X_{1 n} \geq x / \widetilde{m}_{n}\right)\right)^{n} .
$$

Consequently, the d.f. $\mathbf{F}$ of $\mathbf{X}$ equals

$$
\mathbf{F}(x)=1-\lim _{n \rightarrow \infty}\left(P\left(X_{1 n} \geq x / \widetilde{m}_{n}\right)\right)^{n}
$$

and in order to show that the limiting r.v. in (4) exists, it is enough to prove that the limit on the right-hand side of (19) exists.

Case 1: $c=0$. By the law of total probability, from property (2) we get

$$
P\left(X_{1 n} \geq x\right)=\mathcal{L}\left(F_{A} ; x^{r}\right),
$$

where $F_{A}$ is a d.f. of $A_{1}$. Consequently,

$$
\left(P\left(X_{1 n} \geq x / \widetilde{m}_{n}\right)\right)^{n}=\left(\mathcal{L}\left(F_{A} ;\left(x / \widetilde{m}_{n}\right)^{r}\right)\right)^{n} .
$$

On the other hand, by assumption (5),

$$
\lim _{n \rightarrow \infty}\left(\mathcal{L}\left(F_{A} ;\left(x / \widetilde{m}_{n}\right)^{r}\right)\right)^{n}=\mathcal{L}\left(\mathbf{F}_{A} ; x^{r}\right) .
$$

Therefore, the limiting d.f. $\mathbf{F}$ in (19) exists and has the form

$$
\mathbf{F}(x)=1-\mathcal{L}\left(\mathbf{F}_{A} ; x^{r}\right),
$$

which is in agreement with $(7)$ when $c=0$.

Case 2: $c>0$. By the law of total probability, from (2) we get

$$
P\left(X_{1 n} \geq x\right)=\int_{0}^{\infty} \int_{0}^{\infty} \exp \left(-a \min \left(x^{r},(b / c)^{s}\right)\right) d F_{A}(a) d F_{B, n}(b),
$$

where $F_{B, n}(b)$ is the d.f. of $\widetilde{b}_{n}\left(B_{2}^{1} \diamond \ldots \diamond B_{n}^{1}\right)$ (see $\left.(1)\right)$. Therefore by the Fubini theorem we have

$$
\begin{aligned}
P\left(X_{1 n} \geq x / \widetilde{m}_{n}\right)= & \int_{0}^{c\left(x / \tilde{m}_{n}\right)^{r / s}} \mathcal{L}\left(F_{A} ;(b / c)^{s}\right) d F_{B, n}(b) \\
& +\mathcal{L}\left(F_{A} ;\left(x / \widetilde{m}_{n}\right)^{r}\right)\left(1-F_{B, n}\left(c\left(x / \widetilde{m}_{n}\right)^{r / s}\right)\right) .
\end{aligned}
$$

As $F_{B}$, the d.f. of $B_{2}^{1}$, is a continuously differentiable function, $F_{B, n}$ is also continuously differentiable, and for $x>0$ we obtain

$$
\frac{d}{d x} P\left(X_{1 n} \geq x / \widetilde{m}_{n}\right)=\left(1-F_{B, n}\left(c\left(x / \widetilde{m}_{n}\right)^{r / s}\right)\right) \frac{d}{d x} \mathcal{L}\left(F_{A} ;\left(x / \widetilde{m}_{n}\right)^{r}\right) .
$$

(Note that for $x=0$ the term $\frac{d}{d x} \mathcal{L}\left(F_{A} ;\left(x / \widetilde{m}_{n}\right)^{r}\right)$ can be infinite, see (24).) 
From (21) we get

$$
\begin{aligned}
\frac{d}{d x}\left(P\left(X_{1 n} \geq x / \widetilde{m}_{n}\right)\right)^{n}= & n\left(P\left(X_{1 n} \geq x / \widetilde{m}_{n}\right)\right)^{n-1} \\
& \times\left(1-F_{B, n}\left(c\left(x / \widetilde{m}_{n}\right)^{r / s}\right)\right) \frac{d}{d x} \mathcal{L}\left(F_{A} ;\left(x / \widetilde{m}_{n}\right)^{r}\right),
\end{aligned}
$$

which is equivalent to

$$
\text { (22) } \begin{aligned}
\frac{d}{d x} \ln ( & \left.P\left(X_{1 n} \geq x / \widetilde{m}_{n}\right)\right)^{n} \\
= & \left(P\left(X_{1 n} \geq x / \widetilde{m}_{n}\right)\right)^{-1}\left(1-F_{B, n}\left(c\left(x / \widetilde{m}_{n}\right)^{r / s}\right)\right) \\
& \times n \frac{d}{d x} \mathcal{L}\left(F_{A} ;\left(x / \widetilde{m}_{n}\right)^{r}\right) .
\end{aligned}
$$

To prove that the limit in (19) exists let us first show that the right-hand side of (22) has a limit as $n \rightarrow \infty$ :

(i) Since $\min \left(x^{r},(b / c)^{s}\right) \leq x^{r}$, we have

$$
\mathcal{L}\left(F_{A} ;\left(x / \widetilde{m}_{n}\right)^{r}\right) \leq P\left(X_{1 n} \geq x / \widetilde{m}_{n}\right) \leq 1 .
$$

From $(20), \lim _{n \rightarrow \infty} \mathcal{L}\left(F_{A} ;\left(x / \widetilde{m}_{n}\right)^{r}\right)=1$ and so

$$
\lim _{n \rightarrow \infty}\left(P\left(X_{1 n} \geq x / \widetilde{m}_{n}\right)\right)^{-1}=1 .
$$

(ii) It follows from assumption (6) that

$$
\lim _{n \rightarrow \infty} F_{B, n}\left(c\left(x / \widetilde{m}_{n}\right)^{r / s}\right)=\mathbf{F}_{B}\left(c x^{r / s}\right) .
$$

(iii) For any nonnegative r.v. $A$ with d.f. $F_{A}$ it follows from the Lebesgue theorem that

$$
\frac{d}{d x} \mathcal{L}\left(F_{A} ; x\right)=-\int_{0}^{\infty} a e^{-a x} d F_{A}(a),
$$

and we have

$$
\begin{aligned}
\frac{d}{d x}\left(\mathcal{L}\left(F_{A} ;\left(x / \widetilde{m}_{n}\right)^{r}\right)\right)^{n} & =\frac{d}{d x} \mathcal{L}\left(F_{A, n} ; x^{r}\right) \\
& =-r x^{r-1} \int_{0}^{\infty} a e^{-a x^{r}} d F_{A, n}(a),
\end{aligned}
$$

where $F_{A, n}$ is the d.f. of the normalized sum $\left(A_{1}+\ldots+A_{n}\right) / \widetilde{m}_{n}^{r}$. This normalized sum is assumed to tend in distribution to the r.v. A (see (5)), and so for any $x>0$,

$$
\lim _{n \rightarrow \infty} \int_{0}^{\infty} a e^{-a x} d F_{A, n}(a)=\int_{0}^{\infty} a e^{-a x} d \mathbf{F}_{A}(a) .
$$


On the other hand, from (24) we get

$$
-r x^{r-1} \int_{0}^{\infty} a e^{-a x^{r}} d \mathbf{F}_{A}(a)=\frac{d}{d x} \mathcal{L}\left(\mathbf{F}_{A} ; x^{r}\right) .
$$

Therefore from (25)-(27) we obtain

$$
\lim _{n \rightarrow \infty} \frac{d}{d x}\left(\mathcal{L}\left(F_{A} ;\left(x / \widetilde{m}_{n}\right)^{r}\right)\right)^{n}=\frac{d}{d x} \mathcal{L}\left(\mathbf{F}_{A} ; x^{r}\right) .
$$

Since

$$
n \frac{d}{d x} \mathcal{L}\left(F_{A} ;\left(x / \widetilde{m}_{n}\right)^{r}\right)=\left(\mathcal{L}\left(F_{A} ;\left(x / \widetilde{m}_{n}\right)^{r}\right)\right)^{-(n-1)} \frac{d}{d x}\left(\mathcal{L}\left(F_{A} ;\left(x / \widetilde{m}_{n}\right)^{r}\right)\right)^{n}
$$

it follows from (20) and (28) that

$$
\lim _{n \rightarrow \infty} n \frac{d}{d x} \mathcal{L}\left(F_{A} ;\left(x / \widetilde{m}_{n}\right)^{r}\right)=\frac{d}{d x} \ln \mathcal{L}\left(\mathbf{F}_{A} ; x^{r}\right) .
$$

Consequently, the right-hand side of (22) has a limit as $n \rightarrow \infty$, equal to $\left(1-\mathbf{F}_{B}\left(c x^{r / s}\right)\right)(d / d x) \ln \mathcal{L}\left(\mathbf{F}_{A} ; x^{r}\right)$.

Let us now prove that $\lim _{n \rightarrow \infty}\left(P\left(X_{1 n} \geq x / \widetilde{m}_{n}\right)\right)^{n}$ exists. For convenience define $S_{n}(x) \equiv\left(P\left(X_{1 n} \geq x / \widetilde{m}_{n}\right)\right)^{n}$. Let $x, x_{0}>0$. Integrating (22) on the interval $\left[x_{0}, x\right]$ (or $\left.\left[x, x_{0}\right]\right)$ we have

$$
\begin{aligned}
(29) \ln \left(\frac{S_{n}(x)}{S_{n}\left(x_{0}\right)}\right)= & \int_{x_{0}}^{x}\left(P\left(X_{1 n} \geq u / \widetilde{m}_{n}\right)\right)^{-1}\left(1-F_{B, n}\left(c\left(u / \widetilde{m}_{n}\right)^{r / s}\right)\right) \\
& \times n \frac{d}{d u} \ln \mathcal{L}\left(F_{A} ;\left(u / \widetilde{m}_{n}\right)^{r}\right) d u .
\end{aligned}
$$

For any $u \operatorname{such}$ that $\min \left(x, x_{0}\right) \leq u \leq \max \left(x, x_{0}\right)$ we have

$$
\begin{gathered}
0 \leq\left(P\left(X_{1 n} \geq u / \widetilde{m}_{n}\right)\right)^{-1} \leq\left(P\left(X_{1 n} \geq \max \left(x, x_{0}\right) / \widetilde{m}_{n}\right)\right)^{-1}, \\
0 \leq 1-F_{B, n}\left(c\left(u / \widetilde{m}_{n}\right)^{r / s}\right) \leq 1,
\end{gathered}
$$

and from (24),

$$
0 \leq-n \frac{d}{d u} \mathcal{L}\left(F_{A} ;\left(u / \widetilde{m}_{n}\right)^{r}\right) \leq-\left.c_{1} n \frac{d}{d u} \mathcal{L}\left(F_{A} ;\left(u / \widetilde{m}_{n}\right)^{r}\right)\right|_{u=\min \left(x, x_{0}\right)},
$$

where $c_{1}$ is a positive constant. Consequently, we deduce from (29) by means of the Fatou lemma that for any $x, x_{0}>0$ the following limit exists:

$$
\lim _{n \rightarrow \infty} \frac{S_{n}(x)}{S_{n}\left(x_{0}\right)}=\exp \left(\int_{x_{0}}^{x}\left(1-\mathbf{F}_{B}\left(c u^{r / s}\right)\right) \frac{d}{d u} \ln \mathcal{L}\left(\mathbf{F}_{A} ; u^{r}\right) d u\right) .
$$

Let $x_{0}>0$ be fixed. We will evaluate the $\operatorname{limit}_{n \rightarrow \infty} S_{n}\left(x_{0}\right)$. The sequence $S_{n}\left(x_{0}\right)$ is bounded by $(23)$. Hence, there exists a convergent subsequence $S_{n_{k}}\left(x_{0}\right)$ with a limit $S\left(x_{0}\right)$. Moreover, by (20) we have

$$
0<\mathcal{L}\left(\mathbf{F}_{A} ; x_{0}^{r}\right) \leq S\left(x_{0}\right)
$$


Therefore, by (30), for any $x>0$ the limit

$$
\begin{aligned}
S(x) & \equiv \lim _{k \rightarrow \infty} S_{n_{k}}(x) \\
& =S\left(x_{0}\right) \exp \left(\int_{x_{0}}^{x}\left(1-\mathbf{F}_{B}\left(c u^{r / s}\right)\right) \frac{d}{d u} \ln \mathcal{L}\left(\mathbf{F}_{A} ; u^{r}\right) d u\right)
\end{aligned}
$$

also exists. Observe now that taking the limit as $k \rightarrow \infty$ we have, by (23),

$$
\mathcal{L}\left(\mathbf{F}_{A} ; x^{r}\right) \leq S(x) \leq 1
$$

and hence $\lim _{x \rightarrow 0} S(x)=1$. On the other hand, by (31),

$$
\lim _{x \rightarrow 0} S(x)=S\left(x_{0}\right) \exp \left(\int_{x_{0}}^{0}\left(1-\mathbf{F}_{B}\left(c u^{r / s}\right)\right) \frac{d}{d u} \ln \mathcal{L}\left(\mathbf{F}_{A} ; u^{r}\right) d u\right) .
$$

Thus

$$
\lim _{k \rightarrow \infty} S_{n_{k}}\left(x_{0}\right)=S\left(x_{0}\right)=\exp \left(\int_{0}^{x_{0}}\left(1-\mathbf{F}_{B}\left(c u^{r / s}\right)\right) \frac{d}{d u} \ln \mathcal{L}\left(\mathbf{F}_{A} ; u^{r}\right) d u\right) .
$$

Since we can repeat the same arguments for any convergent subsequence of $S_{n}\left(x_{0}\right)$ we see that they all have the same limit and

$$
\lim _{n \rightarrow \infty} S_{n}\left(x_{0}\right)=\exp \left(\int_{0}^{x_{0}}\left(1-\mathbf{F}_{B}\left(c u^{r / s}\right)\right) \frac{d}{d u} \ln \mathcal{L}\left(\mathbf{F}_{A} ; u^{r}\right) d u\right) .
$$

Combining the above result with (30) we conclude that for any $x>0$ we have

$$
\lim _{n \rightarrow \infty} S_{n}(x)=\exp \left(\int_{0}^{x}\left(1-\mathbf{F}_{B}\left(c u^{r / s}\right)\right) \frac{d}{d u} \ln \mathcal{L}\left(\mathbf{F}_{A} ; u^{r}\right) d u\right),
$$

which obviously does not depend on $x_{0}$. Therefore we obtain

$$
\begin{aligned}
\mathbf{F}(x) & =1-\lim _{n \rightarrow \infty}\left(P\left(X_{1 n} \geq x / \widetilde{m}_{n}\right)\right)^{n} \\
& =1-\exp \left(\int_{0}^{x}\left(1-\mathbf{F}_{B}\left(c u^{r / s}\right)\right) \frac{d}{d u} \ln \mathcal{L}\left(\mathbf{F}_{A} ; u^{r}\right) d u\right) .
\end{aligned}
$$

Consequently, the limiting r.v. $\mathbf{X}$ in (4) exists and its d.f. $\mathbf{F}$ has the form (7).

Proof of Theorem 2. The r.v. A (see (5)) is the limit in distribution of the normalized sums of nonnegative i.i.d. r.v.'s. Therefore, from the theory of stable laws, it has to be either $\alpha$-stable (stable with respect to the summation) or degenerate $\left(\mathbf{A}=a_{0}\right.$ with probability 1 , where $a_{0}>0$ under the assumptions of the theorem). Consequently, we have

$$
\frac{d}{d u} \ln \mathcal{L}\left(\mathbf{F}_{A} ; u^{r}\right)=-\alpha r \Lambda_{1}^{\alpha} u^{r \alpha-1}
$$


where $\alpha=1$ and $\Lambda_{1}=a_{0}$ in the degenerate case; otherwise, $0<\alpha<1$ is an index of stability and $\Lambda_{1}$ is a positive constant.

Similarly, the limiting r.v. B (see (6)) has to be either $\diamond$-stable or degenerate $\left(\mathbf{B}=b_{0}\right.$ with probability $\left.1, b_{0}>0\right)$. Therefore, for $c>0$ we deduce from (7) and (32) that in the degenerate case

$$
\mathbf{F}(x)=1-\exp \left(-\left(\Lambda_{1} \min \left(x^{r},\left(b_{0} / c\right)^{s}\right)\right)^{\alpha}\right) .
$$

When $\mathbf{B}$ is nondegenerate it is $\diamond$-stable and its d.f. $\mathbf{F}_{B}$ is a continuous function. Hence, the d.f. $\mathbf{F}$ is differentiable and by (7) and (32) it satisfies the differential equation (9).

When $c=0$ we obtain (8) from (7) and (32).

4. Applications. In the past decade, a considerable attention has been paid to attempts to establish fundamental physical models for the experimentally observed "universal characteristics" in dynamical properties of disordered physical systems such as amorphous semiconductors, insulators, polymers, molecular solid solutions and glasses [Jonscher (1983), Palmer et al. (1984), Montroll and Bendler (1984), Klafter and Shlesinger (1986), Dissado and Hill (1987), Płonka (1991), Scher et al. (1991), Klafter et al. (1992), Hunt (1994)]. From the mathematical point of view, the most important and puzzling problem is to recognize the stochastic dependence between variables describing individual entities constituting the system that leads to the universal characteristics. Inspired by the physical investigations [Klafter and Shlesinger (1986), Płonka (1991), Weron and Jurlewicz (1993)] we present in this section an abstract stochastic description, based on the concept of CED systems, for two examples of disordered materials, hoping that it may be helpful in searching for the solution of the above problem.

EXAMPLE 1 (Dielectric relaxation in dipolar materials). The physical basis for the dielectric response behaviour in the bound dipole class has been the subject of extensive research [Jonscher (1983), Montroll and Bendler (1984), Klafter and Shlesinger (1986), Dissado and Hill (1987), Weron and Weron (1987), Scher et al. (1991), Weron (1991) and Weron (1992)]. It has become clear that the functions which describe the dielectric relaxation in condensed systems deviate considerably from the predictions of the exponential relaxation law [Jonscher (1983), Dissado and Hill (1987)]. On the basis of experimental observations it has been argued that from two types of function proposed, the "stretched exponential" function

$$
\phi(t)=\exp \left(-\left(\omega_{p} t\right)^{1-n}\right)
$$

and the "double-power" type function

$$
-\frac{d \phi(t)}{d t} \propto \begin{cases}\left(\omega_{p} t\right)^{-n} & \text { for } t \ll 1 / \omega_{p}, \\ \left(\omega_{p} t\right)^{-m-1} & \text { for } t \gg 1 / \omega_{p},\end{cases}
$$


the second one fits the observed behaviour better. Here $\phi(t)$ is the so-called relaxation function of the system, $0<n, m<1$, and $\omega_{p}$ is a characteristic constant. The relaxation function $\phi(t)$ expresses the probability that the system, consisting of a large number $N$ of relaxing dipoles, as a whole does not change its initial state up to time $t$, so it has to be given by [Weron (1991)]

$$
\phi(t)=\lim _{N \rightarrow \infty} P\left(\widetilde{a}_{N} \min \left(\theta_{1 N}, \ldots, \theta_{N N}\right) \geq t\right),
$$

where $\widetilde{a}_{N}$ is a suitable normalizing constant, and the r.v. $\theta_{i N}$ is the time needed for changing the initial orientation by the $i$ th dipole, $1 \leq i \leq N$.

Let us find now the direct relation of the concept of the CED system to the dielectric relaxation in dipolar materials. In general, because of the cluster ("defect" region) structure of these materials [Dissado and Hill (1987)], individual dipoles and their local environment do not remain independent during the process of relaxation. In this picture, not every dipole subject to an external field has to change its initial position with probability 1 , even after a very long time. There is a constraint given by the time of structural reorganization of the slowest cluster in the surroundings. In a system consisting of a number $N$ of relaxing dipoles, the probability that the $i$ th dipole has not changed its initial position up to time $t$ given its relaxation rate $\beta_{i}$ and the maximum of the times $\eta_{j}^{i}, j \neq i$, of structural reorganization in all surrounding clusters (under a suitable normalization) is equal to [Weron and Jurlewicz (1993)]

$$
\begin{aligned}
P\left(\theta_{i N} \geq t \mid \beta_{i}=b, \widetilde{b}_{N} \max \left(\eta_{1}^{i}, \ldots, \eta_{i-1}^{i}, \eta_{i+1}^{i}, \ldots,\right.\right. & \left.\left.\eta_{N}^{i}\right)=t^{\prime}\right) \\
& =\exp \left(-b \min \left(t, t^{\prime}\right)\right)
\end{aligned}
$$

for $\widetilde{b}_{N}>0, b, t, t^{\prime} \geq 0$. Hence, the sequence $\theta_{1 N}, \ldots, \theta_{N N}$ is a CED system given $\left\{\beta_{i}\right\}$ and $\left\{\eta_{j}^{i}\right\}$ with the operation $\diamond=\max$ and with parameters $N, \widetilde{b}_{N}, c=1$ and $r=s=1$. Therefore, under the assumptions of Theorems 1 and 2 which are expected to be satisfied from physical considerations, the relaxation function (35) is well defined and has the form $\phi(t)=1-\mathbf{F}(t)$ for $\mathbf{F}(t)$ taken from (7). Moreover, by (9) and (10), it satisfies the differential equation

$$
\frac{d \phi}{d t}(t)=-\alpha \Lambda(\Lambda t)^{\alpha-1}\left(1-\exp \left(-(\Lambda t)^{-\gamma} / k\right)\right) \phi(t) .
$$

Here $\Lambda=\Lambda_{1}$ and $k=\left(\Lambda_{2} / \Lambda_{1}\right)^{\gamma}$. The above equation is called the general relaxation equation [Weron (1992)] and, as shown in [Weron and Jurlewicz (1993)], its solution has the double-power form (34) if and only if $\gamma \geq \alpha$. The exponents $n, m$ of the power law are

$$
n=1-\alpha \quad \text { and } \quad m= \begin{cases}\alpha / k & \text { if } \gamma=\alpha \\ \gamma-\alpha & \text { if } \gamma>\alpha\end{cases}
$$


The CED system idea also yields the stretched exponential form (33) and the conventional exponential form $\phi(t)=\exp (-\Lambda t), \Lambda>0$, of the relaxation function (35). These forms follow from (7) when the parameter $c$ is assumed to be equal to 0 . This corresponds to the case when the cluster structure is neglected and the interpretation of relaxation phenomena is based on the concept of a system of independent exponentially relaxing dipoles. The exponential relaxation, not obeyed by most of the investigated systems, is obtained in the very special case of degenerate $\operatorname{limit} \lim _{N \rightarrow \infty}\left(\beta_{1}+\ldots\right.$ $\left.+\beta_{N}\right) / a_{N}$.

EXAMPle 2 (Nonexponential first-order reactions). Classical chemical kinetics formulated for isolated reactions in homogeneous systems fails to describe experimental data even for elementary reactions at low temperatures or in very short time periods [Płonka (1991)]. Lowering the temperature to slow down the reaction rate and to use the standard spectroscopic techniques one usually vitrifies the system, and in glasses one is forced to deal with the full complexity of the disordered medium.

On the basis of experimental data it has been found [Płonka (1991)] that bimolecular reactions $\mathcal{A}+\mathcal{B} \rightarrow \mathcal{A B}$ are adequately described by kinetic equations for the concentrations $c_{\mathcal{A}}$ and $c_{\mathcal{B}}$ of reacting particles $\mathcal{A}$ and $\mathcal{B}$, respectively, with a time-dependent reaction rate coefficient $k(t)$. Namely,

$$
-\frac{d c_{\mathcal{A}}(t)}{d t}=k(t) c_{\mathcal{A}}(t) c_{\mathcal{B}}(t)
$$

For first-order reactions, i.e., when $c_{\mathcal{B}}(0) \gg c_{\mathcal{A}}(0)$ and hence $c_{\mathcal{B}} \approx$ const, the coefficient $k(t)$ has been observed to be equal to

$$
k(t)=k_{0}\left(t / \tau_{0}\right)^{\alpha-1}, \quad 0<\alpha<1 .
$$

Moreover, the higher the temperature or the longer the time period the less dispersive the reaction in a given system, i.e., the closer to 1 the numerical value of $\alpha$, which corresponds to the classical kinetics with time-independent specific reaction rate [Płonka and Paszkiewicz (1992)].

Integration of $(36)$ with $c_{\mathcal{B}} \approx$ const and the time-dependent coefficient (37) yields

$$
c_{\mathcal{A}}(t) / c_{\mathcal{A}}(0)=\exp \left(-\left(t / \tau_{0}\right)^{\alpha}\right) .
$$

The "stretched exponential" function (33) is then the empirical form of decay laws adequate not only in relaxation phenomena but also in reaction kinetics. The form of $k(t)$, once regarded as empirical, has been shown [Płonka (1991), Płonka and Paszkiewicz (1992)] to follow from reaction modelling in systems with a static disorder which becomes fully evidenced when the reaction rates exceed markedly those of internal rearrangements. However, the universality of the empirical law (37) has not been proved yet. 
It can be shown now that the universal form (37) of the time-dependent reaction rate coefficient $k(t)$ is a consequence of Theorems 1 and 2 for CED systems with parameter $c=0$. Namely, in first-order reactions, where $c_{\mathcal{B}}(0) \gg c_{\mathcal{A}}(0)$, each particle $\mathcal{A}$ has to react with probability 1 after a long enough time and the probability that the life-time $\theta_{i N}$ of the $i$ th $\mathcal{A}$-particle is longer than $t$, given only its reaction constant $\xi_{i}$, decays exponentially:

$$
P\left(\theta_{i N} \geq t \mid \xi_{i}=a\right)=\exp (-a t) .
$$

This means that the sequence $\theta_{1 N}, \ldots, \theta_{N N}$ is a CED system given $\left\{\xi_{i}\right\}$ with parameters $c=0$ and $r=1$. Moreover, $c_{\mathcal{A}}(t) / c_{\mathcal{A}}(0)$ is the probability that the life-time of a system of reactants prepared at $t=0$ is longer than $t$. Under the assumptions of Theorems 1 and 2 which are expected to be satisfied from the chemical point of view, $c_{\mathcal{A}}(t) / c_{\mathcal{A}}(0)$ equals $1-\mathbf{F}(t)$, where $\mathbf{F}(t)$ is given by (8) with $r=1$ and $0<\alpha \leq 1$, which is in agreement with empirical form (38) with $\tau_{0}=1 / \Lambda_{1}$. Consequently, in the case of first-order chemical reactions the required time-dependent form (37) of the reaction rate coefficient $k(t)$ is the only possible one.

Acknowledgments. The authors would like to acknowledge fruitful discussions with Andrew K. Jonscher and Andrzej Płonka.

\section{References}

P. Billingsley (1979), Probability and Measure, Wiley, New York.

L. Breiman (1992), Probability, SIAM, Philadelphia.

L. A. Dissado and R. M. Hill (1987), Self-similarity as a fundamental feature of the regression of fluctuations, Chem. Phys. 111, 193-207.

W. Feller (1966), An Introduction to Probability and Its Applications, Vol. 2, Wiley, New York.

M. R. de la Fuente, M. A. Perez Jubindo and M. J. Tello (1988), Two-level model for the nonexponential Williams-Watts dielectric relaxation, Phys. Rev. B37, 20942101.

A. Hunt (1994), On the "universal" scaling of the dielectric relaxation in dipole liquids and glasses, J. Phys.: Condens. Matter, to appear.

A. Janicki and A. Weron (1994), Can one see $\alpha$-stable variables and processes? Statist. Sci. 9, 109-126.

A. K. Jonscher (1983), Dielectric Relaxation in Solids, Chelsea Dielectrics, London.

J. Klafter and M. F. Shlesinger (1986), On the relationship among three theorems of relaxation in disordered systems, Proc. Nat. Acad. Sci. U.S.A. 83, 848-851.

J. Klafter, M. F. Shlesinger, G. Zumoffen and A. Blumen (1992), Scale invariance in anomalous diffusion, Phil. Mag. B65, 755-765.

M. R. Leadbetter, G. Lindgren and H. Rootzen (1986), Extremes and Related Properties of Random Sequences and Processes, Springer, New York.

S. Mittnik and S. T. Rachev (1991), Modelling asset returns with alternative stable distributions, Stony Brook Working Papers WP-91-05 1-63. 
E. W. Montroll and J. T. Bendler (1984), On Lévy (or stable) distributions and the Williams-Watts model of dielectric relaxation, J. Statist. Phys. 34, 129-162.

R. G. Palmer, D. L. Stein, E. Abrahams and P. W. Anderson (1984), Models of hierarchically constrained dynamics for glassy relaxation, Phys. Rev. Lett. 53, 958961.

A. Płonka (1991), Developments in dispersive kinetics, Prog. Reaction Kinetics 16, 157333.

A. Płonka and A. Paszkiewicz (1992), Kinetics in dynamically disorderd systems: Time scale dependence of reaction patterns in condensed media, J. Chem. Phys. 96, $1128-1133$.

S. T. Rachev (1991), Probability Metrics and the Stability of Stochastic Models, Wiley, Chichester.

H. Scher, M. F. Shlesinger and J. T. Bendler (1991), Time-scale invariance in transport and relaxation, Phys. Today 44, 26-34.

N. G. Van Kampen (1987), Stochastic Processes in Physics and Chemistry, Elsevier, Amsterdam.

K. Weron (1991), A probabilistic mechanism hidden behind the universal power law for dielectric relaxation: General relaxation equation, J. Phys.: Condens. Matter 3, 91519162.

K. Weron (1992), Reply to the comment by A. Hunt, ibid. 4, 10507-10512.

K. Weron and A. Jurlewicz (1993), Two forms of self-similarity as a fundamental feature of the power-law dielectric response, J. Phys. A: Math. Gen. 26, 395-410.

K. Weron and A. Weron (1987), A statistical approach to relaxation in glassy materials, in: Mathematical Statistics and Probability Theory, Vol. B, P. Bauer et al. (eds.). Reidel, 245-254.

V. M. Zolotariev (1986), One-dimensional Stable Distributions, Amer. Math. Soc., Providence.

Agnieszka Jurlewicz and Aleksander Weron Hugo Steinhaus Center for Stochastic Methods

Karina Weron Technical University of Wrocław Institute of Physics 50-370 Wrocław, Poland

\section{Technical University of Wrocław} 50-370 Wrocław, Poland 